\title{
论 文
}

\section{基于相空间重构和Volterra的非线性寒区气温预测 方法}

何鲜峰 ${ }^{1,2 *}$, 汪自力 ${ }^{1,2}$, 何启 $^{3}$, 张宝森 $^{2,4}$

1. 黄河水利委员会黄河水利科学研究院工程力学研究所, 郑州 450003;

2. 水利部堤防安全与病害防治工程技术研究中心, 郑州 450003;

3. 河海大学水利水电学院, 南京 210098 ;

4. 黄河水利委员会黄河水利科学研究院防汛减灾研究所, 郑州 450003

* E-mail: hexianfeng@hky.yrcc.gov.cn

收稿日期: 2018-01-16; 接受日期: 2018-05-16; 网络版发表日期: 2019-03-08

国家重点研发计划项目(编号：2016YFC0401610)、水利公益性行业科研专项经费项目(编号：201401022)和黄河水利科学研究院基本科研业 务费专项项目(编号: HKY-JBYW-2014-04和HKY-JBYW-2017-21)资助

摘要 对寒区冬季气温时间序列的混沌特性及其应用技术进行了研究. 先通过0-1混沌测试法确定寒区冬季气温 时间序列具有混沌特性, 然后通过相空间重构, 分别利用 C-C算法和 G-P算法确定延迟时间和嵌入维数. 在此基础 上，提出了一种相空间重构和Volterra滤波的寒区冬季气温预测方法. 实例分析表明，提出的预测方法在预测精 度、预测误差、预测效果方面均优于常见模型，证明该预测方法是可行和有效的.

关键词 0-1测试, 气温, 时间序列, 混沌

\section{1 引言}

寒区河流经常面临两种洪水风险，即夏季暴雨洪 水和冬季冰凌洪水. 冰凌洪水由于偶发性强、上涨速 度快、历时久，因此对寒区河流、水库冬季运行造成 的威胁和灾害比夏季洪水更大. 研究表明，寒区无论 水库还是河道, 冰凌变化情况受温度影响极大 ${ }^{[1 ~ 3]}$, 所 以, 研究寒区冬季气温变化规律并对其进行预测, 对增 强区域内的河道和水库预警能力，提高安全管理水平 具有重要理论价值和应用价值.

当前气温预测方法可分为基于数理方程的物模仿
真法和时序数据法两大类. 物模仿真法一般基于复杂 的数理方程和必要的边界条件, 通过计算进行气温预 测 ${ }^{[4 ~ 6]}$, 这种预测方法依赖于高精度的计算模型、可靠 的边界条件和大量的计算资源, 目前我国的气温数值 预报模型还比较少, 用于寒区河流、水库冰凌预测的 气温预报数值模型更少. 因此, 基于时序数据的寒区 气温预测研究在实践中具有很大的应用潜力.

国内外许多学者对气温预测问题已进行了广泛研 究, 其中包括AR模型 ${ }^{[2]}$ 、ARMA模型 ${ }^{[7]}$ 等线性模型, 以 及 SVM 模型 ${ }^{[8]}$ 、神经网络模型 ${ }^{[9,10]}$ 、灰色模型 ${ }^{[11]}$ 等非 线性预测模型. 虽然上述模型在多种场合中得到应用,

引用格式: 何鲜峰,汪自力, 何启, 等. 基于相空间重构和Volterra的非线性寒区气温预测方法. 中国科学: 技术科学, 2019, 49: 733-740

He X F, Wang Z L, He Q, et al. The cold regions' temperature nonlinear prediction method basing on phase space reconstruction and Volterra filter (in Chinese). Sci Sin Tech, 2019, 49: 733-740, doi: 10.1360/N092018-00011 
但仍存在许多局限性. 相关研究表明, 气温的变化具有 非平稳特征 ${ }^{[12]}$, 而基于线性回归的 AR 模型和自回归的 ARMA模型虽然具有使用方便、运算量小的优点，但 只适合平稳过程或简单非平稳过程的预测，对于具有 较强非线性特点的寒区气温变化难以描绘其变换过 程, 预测精度有限. 神经网络虽然具有较好的非线性和 自学习能力, 但存在易陷入局部最优、收玫速度慢、 易振荡等问题，且隐含层及层内单元数目难以确定; SVM 具有较好的泛化能力, 能够较好地解决非线性、 高维、局地优势等问题，但其关键参数的笁选依赖于 人工 ${ }^{[13]}$, 对使用者要求较高.

本文针对寒区冬季气温预测问题开展了相关研 究. 首先对寒区冬季气温观测数据进行混沌特性分析, 通过0-1混沌测试方法确定寒区冬季气温时间序列具 有混沌特性. 针对寒区冬季气温时间序列的混沌特性, 通过时间延迟和嵌入维数的确定, 重构相空间, 在此基 础上提出了Volterra多步预测模型．实例分析表明该模 型具有较高的预测精度和适应性，可以为寒区冬季气 温预测提供依据.

\section{2 寒区冬季气温的混沌特性分析}

混沌系统虽然是非线性动力系统中的确定系统, 但其对初始条件有很强的依赖性，因此无法做到一次 建模长久使用，然而系统的确定性，使其在一定范围 内具有预测能力. 因此, 对连续时间序列进行预测前, 首先要判断其是否为混沌序列.

本文采用0-1测试方法 ${ }^{[14 ~ 16]}$ 进行寒区冬季气温时 间序列混沌特性判别. 设寒区冬季气温观测数据为 $x(i), i=1,2, \ldots, N$, 选择 $c$ 为 $(0,2 \pi)$ 的随机数, 定义函数 $p(n), q(n)$ 如下:

$p(n)=\sum_{j=1}^{n} x(j) \cos (\theta(j)), \quad n=1,2, \ldots, N$,

$q(n)=\sum_{j=1}^{n} x(j) \sin (\theta(j)), \quad n=1,2, \ldots, N$,

$\theta(j)=j c+\sum_{i=1}^{j} x(i), \quad j=1,2, \ldots, n$.

基于 $p(n)$ 与 $q(n)$, 定义均方位移 $M(n)$ 为

$M(n)=M_{c}(n)-(E \phi)^{2} \frac{1-\cos n c}{1-\cos c}$,

式中,

$$
\begin{aligned}
M_{c}(n)= & \lim _{N \rightarrow \infty} \frac{1}{N} \sum_{j=1}^{N}\left\{[p(j+n)-p(j)]^{2}\right. \\
& \left.+[q(j+n)-q(j)]^{2}\right\}, \\
M(n)= & M_{c}(n)-(E \phi)^{2} \frac{1-\cos n c}{1-\cos c} .
\end{aligned}
$$

如果函数 $p(n)$ 与 $q(n)$ 的轨迹为布朗运动, 则上面的 函数 $M(n)$ 随时间呈线性增长; 若函数 $p(n)$ 与 $q(n)$ 的轨迹 有界, 则函数 $M(n)$ 也有界. 定义如下增长率 $K_{c}$ 为

$K_{c}=\operatorname{corr}(M(n), n)=\frac{\operatorname{cov}(M(n), n)}{\sqrt{\operatorname{var}(M(n)) \operatorname{var}(n)}}$.

当 $K_{c} \approx 1$ 时，表示该时间序列具有混沌特性；当 $K_{c} \approx 0$ 时， 表示该时间序列不具有混沌特性 ${ }^{[15]}$. $n$ 的取值应满足 $n \leq n_{\mathrm{cut}}<<N$, 一般取 $n_{\mathrm{cut}}=N / 10$ 即可满足要求.

以内蒙古自治区某水文站冬季气温为例 (以下简 称为A水文站)，采集2016年11月5日 2017年3月18日 期间内每隔 $1 \mathrm{~h}$ 的气温数据 2697 组, 变化过程如图 1 所示.

利用0-1混沌测试法, 对2697组气温时间序列进行

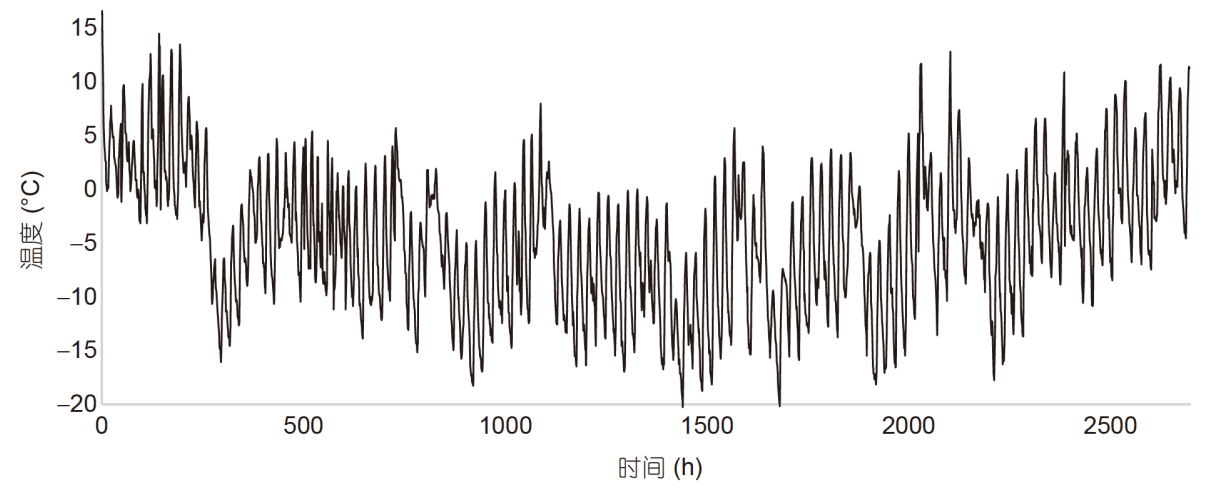

图 1 原始观测序列 
分析, 得到图2所示的 $p(n)-q(n)$ 轨迹图, 该图显示 $p(n)$ 与 $q(n)$ 的运动呈现布朗运动特征. 相应的 $M(n)-n$ 图和 $K_{c}-c$ 图如图3和4所示. 图3表明, $M(n)$ 随 $n$ 呈线性增长, $M(n)$ 值介于 $0 \sim 18000$ 之间, 图4则显示渐进增长率 $K_{c}$ 稳定在 0.996 附近, $K_{c} \approx 1.0$, 表明 $\mathrm{A}$ 水文站冬季气温具有混沌 特性.

\section{3 相空间重构与可预测时间尺度分析}

寒区冬季气温时间序列具有混沌特性, 说明借助 相空间重构可对其进行预测。相空间重构是混沌时间 序列预测的基础，目前一般采用延迟矢量法重构 ${ }^{[17]}$. 该方法的基本观点是：混沌动力系统中各相点随时间 的演化过程是由与其彼此影响的相点决定的，所以这 些相点的讯息就掩盖在相点的进化过程中，重构相点 的轨迹也展现了原系统的发展变化过程.

设 $x(t), t=0,1,2, \ldots, N$ 为寒区冬季气温时间序列, 对 嵌入维 $m$, 可得到一系列 $m$ 维延迟矢量(相点)为

$X\left(t_{i}\right)=\left[x\left(t_{i}\right), x\left(t_{i}+\tau\right), \ldots, x\left(t_{i}+(m-1) \tau\right)\right]$, $i=1,2, \ldots, n$,

式中, $m$ 为嵌入维, $\tau$ 为延迟时间, $n=N-(m-1) \tau$ 表示由 $N$ 个原始观测点重构成 $m$ 维矢量点的数量.

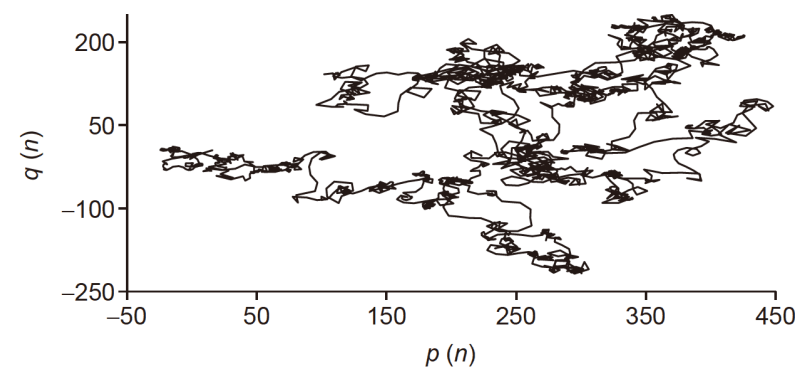

图 $2 p(n)$ 与 $q(n)$ 轨迹图

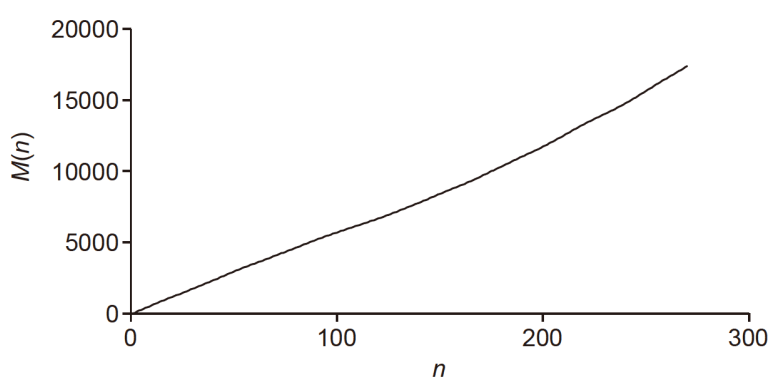

图 3 均方位移 $M(n)$ 变化图

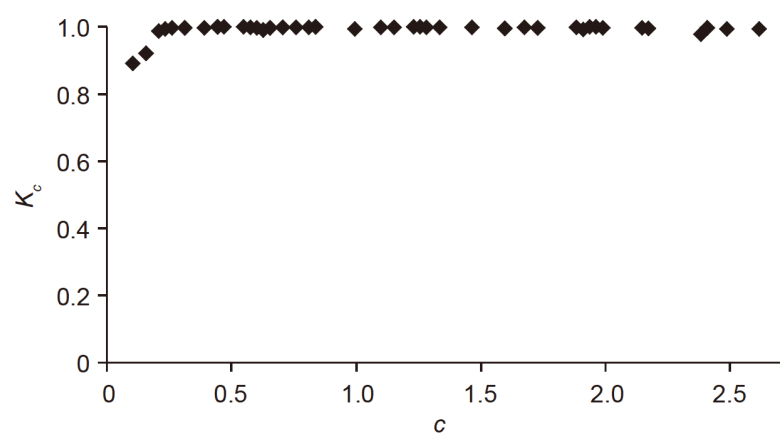

图 4 渐进增长率 $K_{c}$ 变化图

Takens认为, $m$ 和 $\tau$ 是相互独立的，并已证明，当 $m$ 足够大时, 动力系统的重构可以将其许多特性保留下 来，若原系统状态空间的维数为 $D$, 则 $m$ 应满足 $m \geq 2 D$ +1 . 但 $m$ 的取值也不宜过大, 否则需要显著增加数据 量，造成计算工作复杂度上升. 对延迟时间 $\tau$ ，当原始 数据序列有限时, 若 $\tau$ 过小, 会造成重构相点间联系性 增加, 导致隐含特征难以显现; 若 $\tau$ 太大, 又会造成重构 系统失真 ${ }^{[18]}$. 因此, 合理选择 $m$ 和 $\tau$ 值对系统相空间重 构意义重大.

本文利用G-P算法 ${ }^{[19]}$ 来选取嵌入维数 $m$. 该算法依 据关联维 $D$ 随着 $m$ 的递增逐步趋于稳定的原理得到 $m$. 对式(8)给出的重构相点, 首先根据式(9)得到关联函数 $C(r)$ :

$C(r)=\frac{1}{n(n-1)} \sum_{1 \leq i<j \leq n} \theta\left(r-\left\|X\left(t_{i}\right)-X\left(t_{j}\right)\right\|\right)$,

式中, $\|\cdot\|$ 为相点距离, 为简化计算, 通常 取 \|\|$=\max \left|\underset{1 \leq k \leq m}{x_{i k}}-x_{j k}\right| . r$ 为以向量 $X\left(t_{i}\right)$ 或 $X\left(t_{j}\right)$ 为圆心的 半径, 对于某一临界距离 $r>0$, 若 $X\left(t_{i}\right)$ 与 $X\left(t_{j}\right)$ 之间的距 离小于 $r$, 说明这两个向量是有关联的; $\theta(\cdot)$ 为Heaviside函数.

$\theta(t)= \begin{cases}0, & t \leq 0, \\ 1, & t>0 .\end{cases}$

适当调整 $r$ 的取值，在适当区间内 $C(r)$ 与 $r$ 有如下关 联性:

$C(r) \infty r^{D}$,

式中, $D$ 定义为关联维, 并有

$D=\lim _{r \rightarrow 0} \frac{\ln C(r)}{\ln r}$. 
在此基础上, 根据 $m \geq 2 D+1$ 可得到嵌入维 $m$.

对时间延迟 $\tau$ 的选取利用H.S. Kim提出C-C算

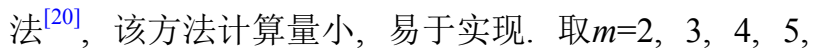
$r_{i}=i \sigma / 2, i=1,2,3,4 . \sigma$ 为时间序列均方差. 计算如下各统 计量 ${ }^{[21]}$ :

$S(t)=\frac{1}{16} \sum_{i=2}^{5} \sum_{j=1}^{4} S\left(m, r_{j}, t\right)$,

$\Delta \bar{S}(t)=\frac{1}{4} \sum_{i=2}^{5} \Delta S(m, t)$,

$S_{\text {cor }}=\Delta S(t)+|S(t)|$,

式中, $\Delta \bar{S}(t), \bar{S}(t)$ 和 $S_{\mathrm{cor}}$ 都可以体现寒区冬季气温时间 序列的自相关性，特别是 $\bar{S}(t)$ 的首次过零点，或 $\Delta \bar{S}(t)$ 的首个局地极小值，或 $S_{\text {cor }}$ 的整体最小值所对应的 $t$ 即 为最优时间延迟 $\tau . S\left(m, r_{j}, t\right), \Delta S(m, t)$ 为C-C算法有关 的统计量 ${ }^{[20]}$.

利用 G-P算法得到前例中寒区冬季气温数据 $\ln C$ $(r)-\ln r$ 的变化曲线如图5所示, 图6为关联维和嵌入维 变化曲线. 由图 5 和 6 可知, 随着嵌入维数 $m$ 的增加, $\ln C$ $(r)-\ln r$ 的线性域内出现饱和现象, 继续增大 $m$, 关联维 数 $D$ 收玫于 5.25 左右, 根据 $m \geq 2 D+1$, 确定 $\mathrm{A}$ 水文站冬季 气温时间序列的嵌入维 $m$ 为 12 .

利用C-C法可得到 $S_{\text {cor }}$ 随时间 $t$ 的变化曲线, 如图7 所示, 从中可以发现, $S_{\mathrm{cor}}$ 的最小值发生在 $t=2$, 故确定 $\mathrm{A}$ 水文站冬季气温间序列的时间延迟 $\tau$ 为 2 . 图 8 为 $\mathrm{A}$ 水 文站冬季气温时间序列的三维相图. 对一个混沌时间 序列而言，若时间延迟取值不当，混沌吸引子的轨迹 会被压缩或折叠，空间点的分布会不均匀，而图8的相 图显示, 当 $\tau=2$ 时, 相空间点的分布区域均匀, 吸引子包 含有系统较多的信息量，因此选择时间延迟 $\tau$ 为 2 是合 理的.

基于上述方式得到的嵌入维和时间延迟，将 $\mathrm{A}$ 水

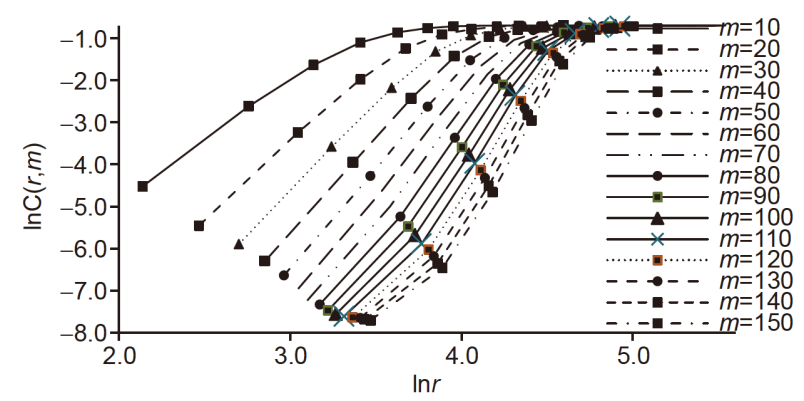

图 $5 \ln C(r)-\ln r$ 变化曲线

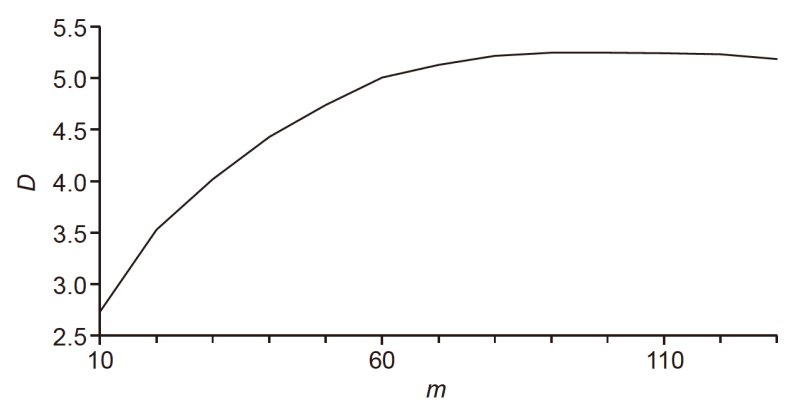

图 $6 D-m$ 变化曲线

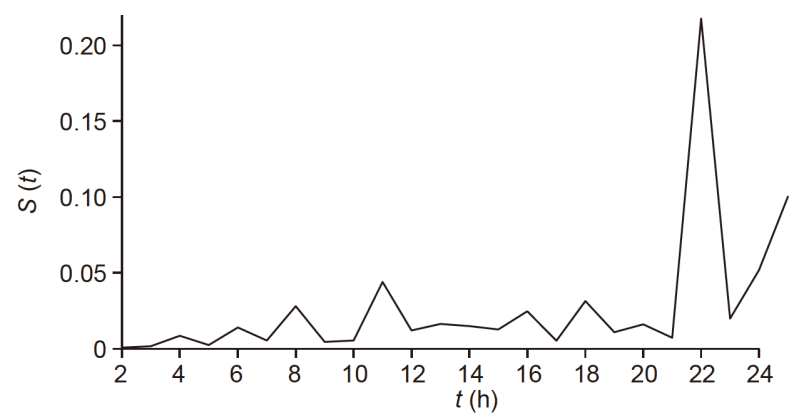

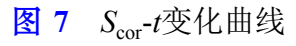

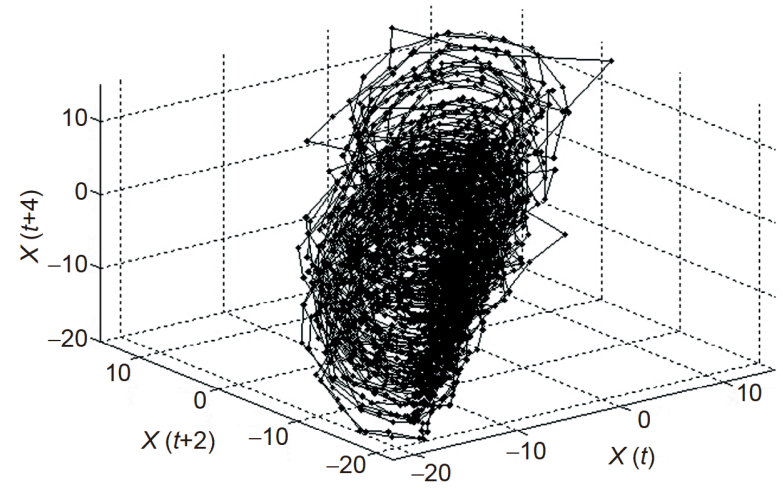

图 $8 x(t)$ 延时三维相图

文站冬季气温观测序列进行相空间重构，采用wolf方 法 ${ }^{[22]}$ 得到Lyapunov指数的最大估计值为 0.0845 , 由此 可以估计 $A$ 水文站冬季气温时间序列最大可预测尺度 为 11 .

\section{Volterra自适应滤波器多步预测模型}

混沌时间序列预测的本质是借助对原始动力系统 的相空间重构，建立与初始系统具有微分同胚吸引子 的低维新系统, 并可由重构系统的当前状态, 推演后 
续时刻的状态，从而实现对原始序列发展趋势的预估. 在各种非线性预测模型中，Volterra模型因其具有所需 训练样本小、易于编程实现等优点，在不同领域被广 泛使用 ${ }^{[23-28]}$. 本文尝试利用Volterra自适应滤波器建立 寒区冬季气温的非线性自适应多步预测模型，并进行 实例验证.

假设离散动力系统的输入为 $X(t)=[x(t), x(t-\tau), \ldots, x$ $(t-(m-1) \tau)]$, 输出为 $y(t)=x(t+1)$, 则此动力系统的Volter$\mathrm{ra}$ 自适应二阶滤波模型为

$$
\begin{aligned}
\widehat{x}(t+1)= & h_{0}+\sum_{i=0}^{m-1} h_{1}(i) x(t-i \tau) \\
& +\sum_{i=0}^{m-1} \sum_{j=i}^{m-1} h_{2}(i, j) x(t-i \tau) x(t-j \tau) .
\end{aligned}
$$

去掉式中第三项则为一阶模型, $\widehat{x}(t+1)$ 为 $x(t+1)$ 的预测 值, 该模型的系数向量和输入向量分别为

$$
\begin{aligned}
& \mathbf{H}(t)= {\left[h_{0}, h_{1}(0), \ldots, h_{1}(m-1), h_{2}(0,0),\right.} \\
&\left.h_{2}(0,1), \ldots, h_{2}(m-1, m-1)\right]^{\mathrm{T}}, \\
& \mathbf{Z}(t)=[ {[1, x(t), x(t-\tau), \ldots, x(t-(m-1) \tau),} \\
&\left.x^{2}(t), x(t) x(t-\tau), \ldots, x^{2}(t-(m-1) \tau)\right]^{\mathrm{T}} .
\end{aligned}
$$

将式(17)和(18)代入式(16), 可得到:

$\widehat{x}(t+1)=\mathbf{H}^{\mathrm{T}}(t) Z(t)$.

上式即为Volterra自适应滤波器的单步预测模型. 若想 进行多步预测, 首先需根据已知样本对滤波器系数进 行训练, 然后利用式(19)进行第一步预测, 得 到 $\widehat{x}(t+1)$, 再将 $\widehat{x}(t+1)$ 加入原始序列，得 到 $X(t+1)=[\widehat{x}(t+1), x(t+1-\tau), \ldots, x(t+1-(m-1) \tau)]$, 进而以 $X(t+1)$ 为系统输入, 得到 $\widehat{x}(t+2)$, 如此反复, 实 现多步预测.

\section{5 预测算例}

\section{1 算例1}

仍以前文内蒙古自治区 $\mathrm{A}$ 水文站冬季气温为例,
把观测到的 2697 组数据中的前 2650 个数据作为训练样 本, 根据前文的Lyapunov指数分析, 该气温系统的预测 步长为 11 , 分别采用本文的Volterra自适应滤波模型 (Volterra adaptive filter model, VAFM)、混沌神经网 络模型(Neural network model, NNM)和自回归滑动平 均模型(auto regressive and moving average model, ARMA)进行预测，3种模型的预测值与实际观测值见 表 1 , 相应的预测值变化曲线和残差曲线分别见图9 和 10 .

\section{2 算例2}

以内蒙古自治区B水文站2015年冬季气温为例, 共取得观测数据1113个, 通过混沌特性分析, 确定其具 有混沌特性，对其进行相空间重构与可测时间尺度分 析, 确定数据重构的嵌入维数 $m$ 为 9 , 时间延迟 $\tau$ 取 2 比 较合适，最大可预测步长为 13 . 利用数据中的前 1100 个数据作为训练样本, 分别采用本文的VAFM模型和 NNM模型、ARMA模型进行预测，3种模型的预测值 与实际观测值详见表 2 , 相应的预测值变化曲线和残 差曲线分别见图11和 12 .

\section{6 不同预测模型适用性分析}

\section{1 预测性能评价指标}

为了对预测模型的预测精度、可靠性和适用性进 行分析, 本文采用常用的 3 种误差评定方法来评价模型 的预测精度.

(1) 绝对平均误差 $e_{\mathrm{NMAE}}$. 绝对平均误差由下式定 义, 该指标用以评价预测值的误差平均幅值:

$e_{\mathrm{NMAE}}=\frac{1}{N} \sum_{i=1}^{N}\left|x_{i}-x_{i}^{\prime}\right|$,

式中, $N$ 为样本容量, $x_{i}$ 为第 $i$ 个观测值, $x_{i}^{\prime}$ 为第 $i$ 个预 测值.

(2) 均方根误差 $e_{\mathrm{NRMAE}}$. 均方根误差可作为模型预

表 1 A 站不同模型预测值比较表

\begin{tabular}{ccccccccccccc}
\hline 序号 & 1 & 2 & 3 & 4 & 5 & 6 & 7 & 8 & 9 & 10 & 11 \\
\hline 实测 & 7.8 & 8.7 & 9.4 & 9.3 & 8.8 & 6.7 & 4.7 & 1.9 & 0.0 & -0.7 & -1.5 \\
NNM模型 & 7.4 & 6.7 & 5.6 & 3.7 & 2.1 & 0.9 & 0.8 & 0.5 & 0.4 & 0.1 & -0.5 \\
ARMA模型 & 8.2 & 9.4 & 10.1 & 10.0 & 10.0 & 8.9 & 7.3 & 5.6 & 4.5 & 4.1 & 4.2 \\
VAFM模型 & 7.2 & 8.2 & 8.1 & 7.3 & 6.1 & 4.2 & 2.5 & 1.6 & 1.1 & 0.7 & 0.2 \\
\hline
\end{tabular}




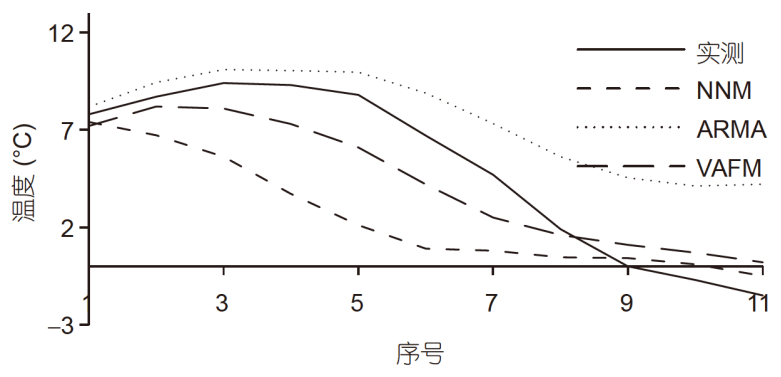

图 $9 \mathrm{~A}$ 站不同模型气温预测曲线

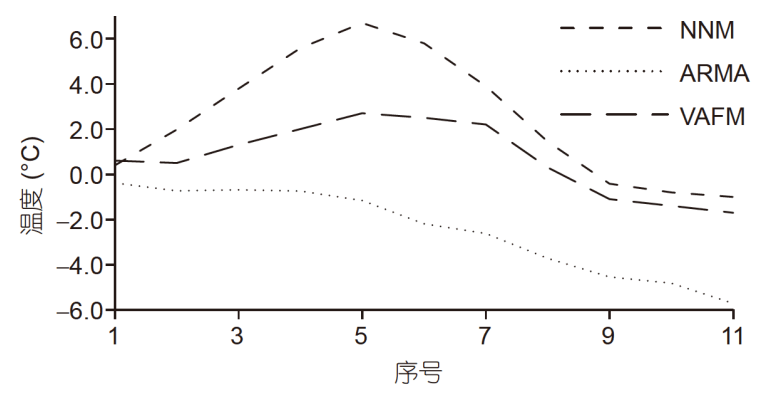

图 10 A站不同模型预测残差

测误差的定量分析指标, 避免正负预测误差相互抵消, 可以评价预测误差的分散程度, 从整体上评价模型预 测系统的性能和预测效果,

$e_{\mathrm{NRMAE}}=\sqrt{\frac{1}{N} \sum_{i=1}^{N}\left(x_{i}-x_{i}^{\prime}\right)^{2}}$.

(3) 方向变差对称值DVS,

$\mathrm{DVS}=\frac{1}{N-1} \sum_{i=1}^{N} \varphi\left(\left(x_{i}-x_{i-1}\right)\left(x_{i}^{\prime}-x_{i-1}^{\prime}\right)\right)$,

式中, $x>0$ 时 $\varphi(x)=1, x \leq 0$ 时 $\varphi(x)=0$.

DVS以百分比形式反映正确预测目标值的变化方 向, 该值越大, 表明预测效果越好.

\section{2 模型适用性评价}

根据算例 1 和算例 2 , 利用上述 3 种常见的预测性能

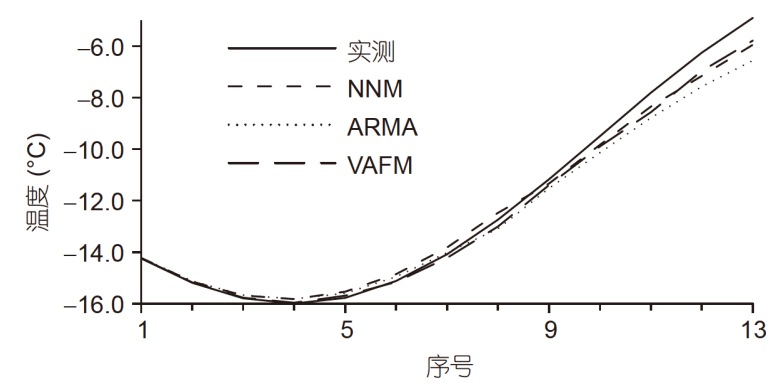

图 11 B站不同模型气温预测曲线

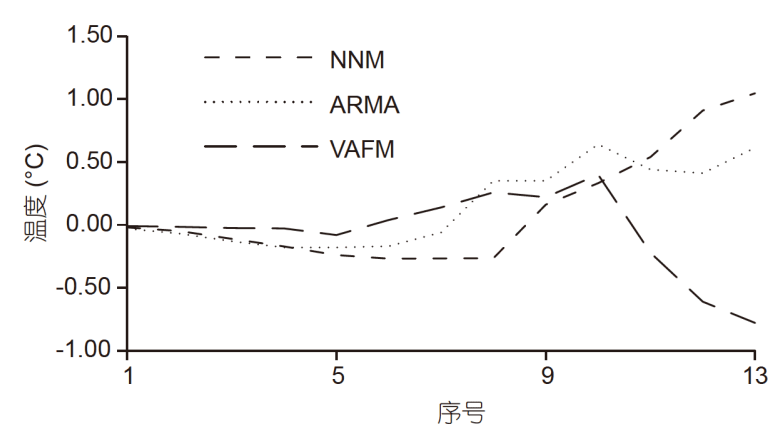

图 12 B站不同模型预测残差

评价指标检验模型的适用性, 成果详见表3.

图9和 11 以及预测模型的方向变差对称值DVS表 明, 3 种模型对气温的变化均具有跟随能力, 但VAFM 模型和ARMA模型均优于NNM模型, 这与NNM模型 存在“过拟合”或“欠拟合”有关. 从图 10,12 和绝对平均 误差 $e_{\mathrm{NMAE}}$ 、均方根误差 $e_{\mathrm{NRMAE}}$ 来看, $\mathrm{VAFM}$ 模型则优 于NNM模型和ARMA模型, 特别是在气温变化存在相 反曲率变化趋势时, VAFM模型在预测精度上的优势 表现更为明显(算例1). 总体而言, VAFM模型无论在 气温预测的跟随能力, 还是在预测精度方面均表现出 较好的稳定性, 可以为寒区冬季气温预测提供参考.

\section{7 结论}

在利用 0-1测试法确定寒区冬季气温变化时间序

表 2 B站不同模型预测值比较表

\begin{tabular}{cccccccccccccc}
\hline 序号 & 1 & 2 & 3 & 4 & 5 & 6 & 7 & 8 & 9 & 10 & 11 & 12 & 13 \\
\hline 实测 & -14.3 & -15.2 & -15.8 & -16.0 & -15.8 & -15.1 & -14.1 & -12.8 & -11.2 & -9.5 & -7.8 & -6.3 & -4.9 \\
NNM模型 & -14.2 & -15.2 & -15.7 & -15.8 & -15.5 & -14.9 & -13.8 & -12.5 & -11.3 & -9.8 & -8.3 & -7.2 & -5.9 \\
ARMA模型 & -14.2 & -15.1 & -15.7 & -15.8 & -15.6 & -15.0 & -14.0 & -13.1 & -11.5 & -10.1 & -8.8 & -7.6 & -6.6 \\
VAFM模型 & -14.2 & -15.2 & -15.8 & -16.0 & -15.7 & -15.2 & -14.2 & -13.0 & -11.4 & -9.9 & -8.6 & -7.0 & -5.8 \\
\hline
\end{tabular}


表 3 不同模型预测误差

\begin{tabular}{|c|c|c|c|c|c|c|}
\hline \multirow{2}{*}{ 模型 } & \multicolumn{3}{|c|}{ 算例1 } & \multicolumn{3}{|c|}{ 算例2 } \\
\hline & $e_{\mathrm{NMAE}}$ & $e_{\mathrm{NRMAE}}$ & DVS (\%) & $e_{\mathrm{NMAE}}$ & $e_{\text {NRMAE }}$ & DVS (\%) \\
\hline NNM模型 & 2.89 & 3.66 & 73 & 0.34 & 0.45 & 100 \\
\hline ARMA模型 & 2.48 & 3.09 & 82 & 0.28 & 0.34 & 100 \\
\hline VAFM模型 & 1.48 & 1.67 & 82 & 0.22 & 0.32 & 100 \\
\hline
\end{tabular}

列具有混沌特性的基础上, 分别采用C-C算法和 G-P算 法得到寒区冬季气温变化时间序列的相空间重构时间 延迟和嵌入维数, 并通过最大Lyapunov指数, 估计了重 构相空间的最大预测尺度. 基于重构相空间, 利用Vol- terra滤波器，构建了寒区冬季气温变化的非线性自适 应多步预测模型. 预测结果显示, Volterra非线性自适 应多步预测模型能够比较准确地预测寒区冬季气温变 化趋势, 并具有较高的预测精度.

\section{参考文献}

1 肖建民, 金龙海, 谢永刚, 等. 寒区水库冰盖形成与消融机理分析. 水利学报, 2004, 6: 80-85

2 乔继平, 王富强, 代俊峰. 黄河宁蒙河段封开河日期预报方法研究. 人民黄河, 2013, 35: 6-7

3 黄文峰, 李志军, 贾青, 等. 水库冰表层形变的现场观测与分析. 水利学报, 2016, 12: 1585-1592

4 贾丽伟, 李维京, 陈德亮, 等. 东北地区月平均大气环流型与哈尔滨气候关系的初步研究. 气象学报, 2006, 64: 236-245

5 金秀良, 宋燕, 吴洪, 等. 应用北半球大气环流系统预测中国降水量和气温. 气象科技, 2014, 42: 1028-1038

6 凌志明, 王毅刚, 陶玲君. 基于OGRE的实时光照及降雨天气仿真. 杭州电子科技大学学报(自然科学版), 2015, 35: 31-35

7 从凌博, 蔡吉花. ARMA模型在哈尔滨气温预测中的应用. 数学的实践与认识, 2012, 42: 190-195

8 蔡吉花, 张世军, 杨丽. EMD-SVM在南京市月平均气温预测中的应用. 数学的实践与认识, 2014, 22: 103-111

9 冀鸿兰, 朝伦巴根, 陈守显. 模糊优选神经网络BP模型在黄河内蒙段封开河预报中的应用. 水利水电科技进展, 2008, 28: 70-72

10 金志凤, 符国槐, 黄海静, 等. 基于BP神经网络的杨梅大棚内气温预测模型研究. 中国农业气象, 2011, 3: 362-367

11 周华任, 李浩然, 孙学金, 等. 一种基于季节指数的灰色马尔科夫气温预测模型. 数学的实践与认识, 2016, 4: 167-173

12 罗妤, 吴坚, 徐金晶. 合肥市平均气温动态变化的非平稳时序分析. 安徽农业大学学报, 2013, 40: 1059-1062

13 唐舟进, 任峰, 彭涛, 等. 基于迭代误差补偿的混沌时间序列最小二乘支持向量机预测算法. 物理学报, 2014, 63: 78-87

14 Gottwald G A, Melbourne I. A new test for chaos in deterministic systems. Proc R Soc A-Math Phys Eng Sci, 2004, 460: 603-611

15 Gottwald G A, Melbourne I. On the implementation of the 0-1 test for chaos. SIAM J Appl Dyn Syst, 2009, 8: 129-145

16 李新杰, 胡铁松, 郭旭宁, 等. 0-1测试方法的径流时间序列混沌特性应用. 水科学进展, 2012, 6: 861-868

17 Takens F. Detecting strange attractors in turbulence. In: Rand D, Young LS, Eds. Dynamical Systems and Turbulence, Warwick 1980. Lecture Notes in Mathematics, Vol 898. Berlin, Heidelberg: Springer, 1981. 366-381

18 汪树玉, 刘国华, 杜王盖, 等. 大坝观测数据序列中的混沌现象. 水利学报, 1999, 7: 23-27

19 Grassberger P, Procaccia I. Measuring the strangeness of strange attractors. Physica D, 1983, 9: 189-208

20 Kim H S, Eykholt R, Salas J D. Nonlinear dynamics, delay times, and embedding windows. Physica D, 1999, 127: 48-60

21 吕金虎, 陆君安, 陈士华. 混沌时间序列分析及其应用. 武汉: 武汉大学出版社, 2005. 237

22 Wolf A, Swift J B, Swinney H L, et al. Determining Lyapunov exponents from a time series. Physica D, 1985, 16: 285-317

23 H. Kashani M, Ghorbani M A, Dinpashoh Y, et al. Integration of Volterra model with artificial neural networks for rainfall-runoff simulation in forested catchment of northern Iran. J Hydrol, 2016, 540: 340-354

24 Zhang B, Billings S A. Volterra series truncation and kernel estimation of nonlinear systems in the frequency domain. Mech Syst Signal Pr, 2017, 84: $39-57$ 


\title{
The cold regions' temperature nonlinear prediction method basing on phase space reconstruction and Volterra filter
}

\author{
HE XianFeng ${ }^{1,2}$, WANG ZiLi ${ }^{1,2}, \mathrm{HE} \mathrm{Qi}^{3}$ \& ZHANG BaoSen ${ }^{2,4}$ \\ ${ }^{1}$ Department of Civil Engineering Mechanics, Yellow River Institute of Hydraulic Research, Zhengzhou 450003, China; \\ ${ }^{2}$ Center for Levee Safety and Disease Prevention Engineering and Technology Research, Ministry of Water Resources, Zhengzhou 450003, China; \\ ${ }^{3}$ College of Water Conservancy and Hydropower Engineering, Hohai University, Nanjing 210098, China; \\ ${ }^{4}$ Department of Disaster Prevention and Mitigation, Yellow River Institute of Hydraulic Research, Zhengzhou 450003, China
}

The chaos characteristics of temperature time series in cold regions' winter were studied. Firstly, it is determined by 0-1 time series of chaotic test method that the chaos characteristics of temperature in cold regions' winter. Secondly, reconstructing phase spaces, and using the C-C algorithm and G-P algorithm to determine the delay time and embedding dimension. Then, based on phase space reconstruction and Vinterra filter, a temperature prediction method in cold regions' winter is proposed. Case analysis shows that the proposed method is superior to the common model in accuracy, prediction error and effect, and the prediction method is feasible and effective.

\section{0-1 test, temperature, time series, chaos}

doi: $10.1360 /$ N092018-00011 